\title{
One-Way Supercurrent Controlled by Magnetic Field
}

\author{
Observation of superconducting diode effect \\ Authors: Fuyuki Ando, Yuta Miyasaka, Tian Li, Jun Ishizuka, Tomonori Arakawa, \\ Yoichi Shiota, Takahiro Moriyama, Youichi Yanase, Teruo Ono \\ Nature, 584, 373 (2020).
}

\section{Recommended with a Commentary by Liang Fu, Massachusetts Institute of Technology}

Nonreciprocal charge transport in electrical conductors is characterized by the dependence of current-voltage characteristic on the direction of current, such that $I(-V) \neq-I(V)$. This behavior is found in semiconductor $p-n$ junctions: due to the built-in potential across the depletion region the resistance is low under forward bias and high under reverse bias. In recent years, another type of nonreciprocal transport has been observed in junction-free bulk crystals under magnetic field, which takes the form of current-dependent resistance $R=R_{0}(1+\gamma B I)[1]$. This so-called magnetochiral effect is allowed in crystals lacking inversion symmetry. It can arise from the combination of spin-orbit and Zeeman splitting that removes the degeneracy between left- and right-moving charge carriers. The resulting nonreciprocal resistance $\gamma$ depends crucially on the relative orientation of the current with respect to the magnetic and spin-orbit fields, see for example Ref.[2]. Since both Zeeman and spin-orbit energies are much smaller than Fermi energy, the nonreciprocal effect is generally weak in normal conductors.

Now, the highlighted work by Ando et al observed for the first time nonreciprocity in the critical current of a superconductor. The system is an artificially designed superconductor film under a small in-plane magnetic field. The film consists of thin alternating layers of three superconducting elements - niobium, vanadium and tantalum, and shows a sharp superconducting at $T_{c}=4.41 \mathrm{~K}$. The authors performed direct-current measurements along the film's plane. Remarkably, they found that the critical current $I_{x}$ along the axis orthogonal to the magnetic field $B_{y}$ is direction dependent, i.e., $I_{c}^{+} \neq I_{c}^{-}$. As a result, a DC current in the range $I_{c}^{-}<I_{x}<I_{c}^{+}$flows without resistance in one direction only, while passing the same current in the opposite direction causes finite resistance, see Fig.1. Thus the junctionfree superconductor film operates as a supercurrent diode and achieves one-way transport of electrical charge without power consumption.

Such supercurrent diode could be useful in superconducting devices and circuits that have practical applications. It may be integrated with SQUID for ultrasensitive magnetic field measurements with ultralow power consumption, or enable signal isolation in superconducting neural networks. Besides its potential for device applications, the highlighted work 

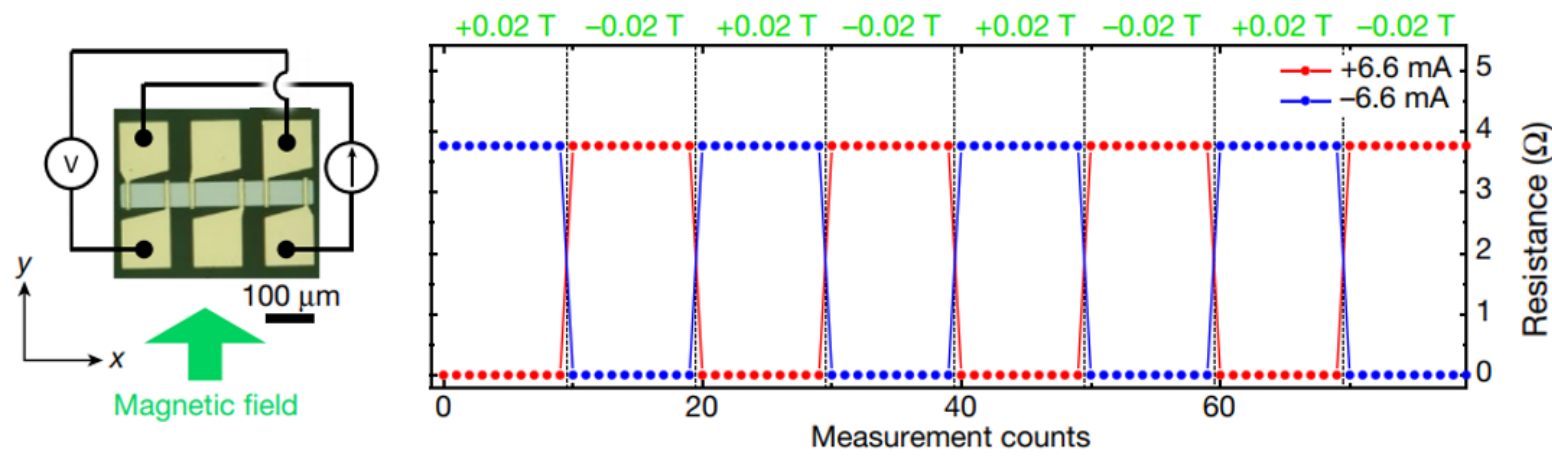

Figure 1: A superconductor film under a small magnetic field $B_{y}=+0.02 \mathrm{~T}$ shows zeroresistance when a current of $6.6 \mathrm{~mA}$ is passed along $+x$ direction, but not $-x$. The superconducting diode effect is switched at $B_{y}=-0.02 \mathrm{~T}$. Taken from the highlighted work.

reveals a previously unknown property of noncentrosymmetric superconductors, and calls attention to non-reciprocal superconducting phenomena in general.

Clearly nonreciprocal critical current $\Delta I_{c} \equiv I_{c}^{+}-I_{c}^{-} \neq 0$ is only allowed in the absence of any symmetry relating $I$ and $-I$. In the experimental setup, the ABC-stacked superlattice structure breaks spatial inversion $(x, y, z) \rightarrow(-x,-y,-z)$. The magnetic field $B_{y}$ (a pseudovector) breaks reflection $x \rightarrow-x$ as well as time-reversal symmetry. The measured $\Delta I$ indeed shows antisymmetric behavior with respect to $B_{y}$. It is important to note that time-reversal symmetry breaking is necessary for direction dependent critical current in a superconductor (at least at $T=0$ ), because otherwise the quantum ground state carrying a dissipationless current would be degenerate with its time-reversed partner carrying the opposite current. In contrast, dissipative charge transport through normal conductors without inversion symmetry can be nonreciprocal at zero magnetic field [3], as predicted in topological insulators and graphene systems [4].

In this experiment on $\mathrm{Nb}-\mathrm{V}$-Ta film, $\Delta I_{c}$ depends on temperature and magnetic field in a complex manner. At $4.2 \mathrm{~K}, \Delta I_{c}$ quickly rises to the maximum value of about $0.3 \mathrm{~mA}-$ which is $\sim 4.5 \%$ of $I_{c}$ - at a very small magnetic field of $0.02 \mathrm{~T}$, then decreases and eventually vanishes as $B_{y}$ increases towards the upper critical field $0.2 \mathrm{~T}$. While the Zeeman effect might be relevant at relatively high magnetic field, it is probably not strong enough to induce the large $\Delta I_{c}$ at such small field as $0.02 \mathrm{~T}$. Since the superconductor film under study is relatively thick $(120 \mathrm{~nm})$, the orbital effect of parallel magnetic field is likely more important.

As far as I know there is no microscopic theory for nonreciprocal critical current in any superconductor. Here I suggest a simple mechanism that may apply to the experiment by Ando et al. First, consider a current-carrying superconducting state in the absence of magnetic field. Due to the broken inversion symmetry of the superlattice, the top and bottom surfaces are non-equivalent, and correspondingly, the current densities at the two surfaces are unequal $j_{1} \neq j_{2}$. The asymmetric surface currents produce a bulk magnetization $M_{y}=\int d z \partial_{z} j_{x}=j_{1}-j_{2}$, in proportional to the transport current $I_{x}$ passing through the film. A parallel magnetic field couples linearly to $M_{y}$ and hence $I_{x}$. Therefore, at finite field the free energies of the superconducting states with current $I_{x}$ and $-I_{x}$ are different, which naturally results in direction dependent critical current. 
The orbital effect of the parallel magnetic field will be suppressed when the thickness of the superconductor film is reduced below the coherence length. It will be very interesting to study in future nonreciprocal effects in two-dimensional superconductors, where direction dependent critical current may come intrinsically from Zeeman and spin-orbit splitting. Recent theory predicts that superconducting fluctuation greatly enhances magnetochiral effect in the normal state above $T_{c}$ [5], which has been observed in 2D superconductors $\mathrm{MoS}_{2}$ [6] and $\mathrm{SrTiO}_{3}[7,8]$. Another direction is to search for nonreciprocal effects in chiral superconductors in the absence of external magnetic field. The highlighted work will surely stimulate a lot of activities in superconductivity research.

\section{References}

[1] Y. Tokura and N. Nagaosa, Nature Communications, 9, 3740 (2018).

[2] Pan He, Steven S.-L. Zhang, Dapeng Zhu, Yang Liu, Yi Wang, Jiawei Yu, Giovanni Vignale and Hyunsoo Yang Nature Physics, 14, 495 (2018).

[3] V. I. Belinicher and B. I. Sturman, Sov. Phys. Usp. 23, 199 (1980).

[4] H. Isobe, S. Y. Xu and L. Fu, Science Advances, 6, eaay2497 (2020).

[5] Ryohei Wakatsuki and Naoto Nagaosa, Phys. Rev. Lett. 121, 026601 (2018).

[6] Ryohei Wakatsuki, Yu Saito, Shintaro Hoshino, Yuki M. Itahashi, Toshiya Ideue, Motohiko Ezawa, Yoshihiro Iwasa and Naoto Nagaosa, Science Advances, 3, e1602390 (2017).

[7] Timo Schumann, Luca Galletti, Hanbyeol Jeong, Kaveh Ahadi, William M. Strickland, Salva Salmani-Rezaie, and Susanne Stemmer, Phys. Rev. B 101, 100503 (2020).

[8] Yuki M. Itahashi, Toshiya Ideue, Yu Saito, Sunao Shimizu, Takumi Ouchi, Tsutomu Nojima and Yoshihiro Iwasa, Science Advances, 6, eaay9120 (2020). 